Cahiers de recherches médiévales

\title{
La Vie du terrible Robert le dyable
}

Un exemple de mise en prose (1496)

\section{Élisabeth Gaucher}

\section{(2) OpenEdition}

\section{Journals}

Édition électronique

URL : https://journals.openedition.org/crm/1472

DOI : $10.4000 / \mathrm{crm} .1472$

ISSN : 1955-2424

Éditeur

Honoré Champion

\section{Édition imprimée}

Date de publication : 30 octobre 1998

Pagination : 153-164

ISSN : 1272-9752

\section{Référence électronique}

Élisabeth Gaucher, « La Vie du terrible Robert le dyable », Cahiers de recherches médiévales [En ligne], 5 | 1998, mis en ligne le 01 octobre 2007, consulté le 15 décembre 2022. URL : http:// journals.openedition.org/crm/1472; DOI : https://doi.org/10.4000/crm.1472

Ce document a été généré automatiquement le 15 décembre 2022.

Tous droits réservés 


\title{
La Vie du terrible Robert le dyable
}

\author{
Un exemple de mise en prose (1496)
}

\author{
Élisabeth Gaucher
}

1 La légende de Robert le diable a connu un succès continu, depuis le roman en vers octosyllabiques qu'en a écrit un auteur anonyme à la fin du XII ${ }^{\mathrm{e}}$ ou au début du XIII ${ }^{e}$ siècle. Elle figure, transcrite en latin, dans le recueil d'exempla d'Étienne de Bourbon (entre 1250 et 1261); elle sert de mythe fondateur au début des Chroniques de Normandie (fin XIII ${ }^{\mathrm{e}}$-début XIV ${ }^{\mathrm{e}}$ siècle); elle a été adaptée pour la scène dans le trentetroisième Miracle de Nostre-Dame par personnages (1375) ; enfin, un Dit de deux-centcinquante-quatre quatrains monorimes, datant de la première moitié du XIV e siècle, en condense la matière en vue d'une diffusion orale et à des fins didactiques ${ }^{1}$.

Cette dernière réécriture a fait l'objet d'une mise en prose, que nous possédons sous la forme d'un incunable lyonnais de quarante-quatre folios, portant la date du 7 mai 1496 : la Vie du terrible Robert le dyable ${ }^{2}$. C'est la matrice d'une longue postérité, les éditions se multipliant à Lyon, Paris et Rouen, tandis que la légende pénètre dans les couches populaires par l'intermédiaire des livrets de la Bibliothèque Bleue. On connaît le rôle décisif joué par la ville de Lyon dans l'essor de l'imprimerie française, qu'elle favorisa grâce à son dynamisme économique. Le roman de Robert le diable était initialement réservé à la noblesse, comme en témoigne le soin apporté à la confection de ses deux manuscrits enluminés ${ }^{3}$. Avec le Dit, écrit par un clerc mais diffusé par des jongleurs pour l'édification du peuple et des bourgeois, commence le processus de vulgarisation ${ }^{4}$. Si l'ouvrage publié en 1496 s'adresse encore à des ecclésiastiques et à des notables, essentiellement hommes d'affaires ou officiers de justice, il semble avoir touché aussi un public d'artisans et de marchands, les romans d'aventures commençant à apparaître démodés dans les milieux lettrés ${ }^{5}$.

3 La teneur du récit frappe, en effet, par sa simplicité. Le duc et la duchesse de Normandie, las d'adresser en vain leurs prières à Dieu, qui leur refuse des enfants, invoquent le diable et voient leur attente comblée par la naissance d'un fils. Mais Robert, dès son plus jeune Age, manifeste une cruauté sanguinaire et s'associe à des brigands pour mettre le duché à feu et à sang. Son dernier crime, qui coûte la vie à sept ermites, lui fait soudain prendre conscience du mal qui le consume. La duchesse révèle 
alors à son fils le secret de sa naissance. Robert, après avoir tenté sans succès de convertir ses compagnons, les extermine et va à Rome, où le pape l'envoie à un ermite, qui lui dicte les clauses de sa pénitence : feindre la folie, la mutité, et disputer sa nourriture aux chiens. Réfugié dans le palais de l'empereur, où il fait office de bouffon, Robert participe incognito, grâce à l'assistance secrète d'un ange qui lui apporte une armure, à la guerre contre les sarrasins et contribue à les repousser victorieusement, malgré une blessure à la jambe. Pour récompenser son mystérieux sauveur, l'empereur de Rome l'invite à se présenter devant la cour afin d'y recevoir sa couronne et la main de sa fille. Épris de celle-ci, mais voyant ses avances repoussées, le sénéchal tente alors d'usurper la gloire de Robert. Un miracle permet à la princesse, muette de naissance, de recouvrer l'usage de la parole et de révéler les mérites du fou, dont elle a découvert le subterfuge. Robert est alors délivré de sa pénitence et, après avoir décliné sa véritable identité, épouse la jeune fille. Il rentre avec elle en Normandie, où il sauve sa mère, devenue veuve, de l'emprise d'un seigneur arrogant. Il regagne Rome pour y venger le meurtre de son beau-père, en tuant le sénéchal félon. Ses jours s'achèvent, irréprochables, dans ses possessions normandes, à la tête desquelles il laisse un fils, Richard.

4 La mise en prose du Dit répond à un souci pragmatique, visant à privilégier le didactisme du message et à satisfaire les nouvelles conditions de réception imposées par la lecture silencieuse. Le style s'adapte à la mode du temps, par la tendance à l'amplification et au formalisme. Quant au remaniement du dispositif narratif, il passe par des opérations de rajeunissement, une plus grande attention portée à la psychologie des personnages, et un souci d'affiner la logique du texte, au nom du principe de la vraisemblance.

5 Les adaptations en prose voient le jour au XIII ${ }^{e}$ siècle, comme en témoignent les œuvres majeures du Lancelot et du Tristan. L'extension de la prose, qui s'accélère en Moyen Français, ne touche pas seulement les anciens textes, qu'elle s'applique à mettre aux goûts d'un public nouveau; elle caractérise aussi les productions inédites. Ce syncrétisme remet en cause les frontières génériques : désormais, les genres narratifs, chansons de geste, romans et récits historiographiques, se confondent sous une forme unique, oblitérant la distinction entre fiction et histoire. L'écriture en prose renvoie aux mécanismes spontanés du langage ordinaire, et s'oppose aux artifices de la rime; d'où l'association du vers à la fable mensongère, tandis que la prose fonctionne comme un label d'authenticité. Le dérimage contribue à donner au texte-source une coloration historique, proche du réel, même si la matière puise dans une tradition imaginaire.

6 Le remanieur qui, en 1496, réécrit le Dit de Robert le diable semble guidé par les même motivations que les autres dérimeurs de la fin du Moyen Âge, bien qu'il n'ait pas jugé nécessaire de les formuler, comme c'est le cas d'ordinaire, dans une préface justifiant son entreprise. L'enjeu didactique prédomine nettement. Dès le prologue, la citation d'autorités reconnues (Boèce, saint Augustin, fol.1) garantit la valeur éthique de son message, qu'il place sous les auspices de Dieu et de la Vierge Marie. Pour souligner le sérieux de son travail, il évoque, sans préciser, «l'istoire» dont il s'inspire (fol. 40). Le récit s'ouvre sur une formule aléthique : « or est vray que... » (fol. 2); un peu plus loin, le remanieur en appelle à la confiance de ses lecteurs : "et ainsi le devez vous croire" (fol.11). Étranger aux règles de virtuosité qui fondent, pour une bonne part, les versions rimées, le texte en prose privilégie l'enseignement de ses destinataires. Il en ressort un constant souci de clarté, visible dans l'architecture narrative. 
7 L'application du principe de la manifestatio aboutit à une logique visuelle propre aux éditions imprimées, que matérialisent l'apparition d'un titre et la subdivision en rubriques. La formule liminaire semble choisie à la fois pour attiser la curiosité et pour établir un contrat de fiabilité quant au genre auquel se rattache le récit: «Vie du terrible Robert le dyable». Le public comprend ainsi qu'il a affaire à une biographie. Le terme «roman» est soigneusement écarté, au profit d'appellations plus scientifiques, «hystoire », «traicté » ou tout simplement « livre» (fol. 1). Les rubriques, quant à elles, servent à indiquer le contenu des chapitres. Elles en résument l'essentiel et annoncent, par avance, les orientations du récit. Parfois leur liste, établie au début de l'œuvre, constitue la table des matières; ce n'est pas le cas ici, et il ne semble pas que la disparition d'un folio ait rendu le volume acéphale. Pour renforcer cette charpente logique, des formules de liaison relient les chapitres entre eux, récapitulant l'épisode que l'on quitte et enchaînant avec le suivant ; ainsi après une victoire remportée contre les sarrasins :

Quant le champ et l'onneur de la journée fut ainsi demouré a l'empereur par l'ayde de Robert, tout armé sus son cheval [il] s'en retourna... (fol. 29).

Ce balisage constant de la narration entraîne la disparition de tout effet de surprise, le lecteur étant guidé, dès le commencement, dans la démonstration des principes théoriques énoncés dans le prologue, à propos des bienfaits de la pénitence.

Contrairement au récit en vers, allusif et discontinu, l'écriture en prose repose sur une explicitation systématique du message qu'elle entend délivrer. Cette distinction recoupe celle qui sépare la diffusion orale et la transmission écrite. L'oralité passe par la stéréotypie, elle fait appel à la connivence du lecteur par des emprunts à une tradition commune, ancrée dans la mémoire culturelle. En revanche, l'écrit atteste la volonté de tout expliquer, conformément à une logique infaillible. Si le texte récité publiquement fondait sa cohérence dans l'intonation et la gestuelle du jongleur, il n'en est plus de même pour la nouvelle littérature, qui fait l'objet d'une lecture individuelle et silencieuse: adaptée à ce nouveau mode de consommation littéraire, la prose construit son propre système de repérage, destiné à supprimer tout ambiguïté et toute rupture dans l'exposé. La Vie du terrible Robert le dyable accuse la tendance, particulièrement nette au $\mathrm{XV}^{\mathrm{e}}$ siècle, à marquer le commencement et la fin de la narration par des formules d'avertissement : «Cy commence » (fol. 1), «Pour mettre fin a nostre present livre» (fol. 44). Cette habitude provient des genres didactiques et témoigne d'un souci d'efficacité pédagogique. Pourtant, la nécessité de guider l'auditoire semble moins nécessaire qu'aux temps de la transmission orale; la lecture est soumise à un contrôle aussi vétilleux qu'autrefois l'écoute.

En 1496, en effet, la lecture privée est établie dans les usages. Notre incunable multiplie les formules qui traduisent la destinée de l'œuvre : «par la lecture d'ycelle [ystoire] », «tous ceulx qui [...] liront ce present livre» (fol. 1)... Mais si le verbe "veoir» traduit cette approche personnelle du récit («comme apres verrés», fol. 5, 13), il n'élimine pas pour autant le verbe « oïr » (« comme avés ouy », fol. 24, 27, 28, 34 ; « vous orrez parler », fol. 1, 34), qui hérite des anciens appels à l'auditoire. Le remanieur recourt parfois aux formules épiques, où le public est invité à imaginer le spectacle raconté, en particulier lors des brillants assauts menés par Robert contre les sarrasins : «la veïssiés » (fol. 29, 32 ; cf. fol. 14), «c'estoit ung plaisir a les regarder » (fol. 32). Ces imitations de l'ancienne récitation orale confèrent au texte une coloration passéiste, auréolée du prestige d'une 
époque révolue, qui suscite la nostalgie. Le dérimeur n'hésite pas à se comparer à un jongleur déclamant son texte devant une assemblée à laquelle il s'associe volontiers : «nous laisserons le dueil de la jeune duchesse et parlerons de Robert» (fol. 44, cf. fol. 36). La solidarité du récitant et de son public, leur proximité vivante et chaleureuse, apparaissent, aux yeux de l'écrivain confronté à la solitude et à l'anonymat, comme un idéal perdu.

Les commentaires restent alors pour lui un moyen d'affirmer son autorité et de rétablir un contact avec ses destinataires. Ils traduisent un penchant moralisateur, non dénué de lourdeur. Plusieurs proverbes, déjà présents dans le Dit, ponctuent les temps forts du récit, auquel l'auteur s'efforce de les adapter avec soin ; ainsi, à propos des prétentions du sénéchal félon:

on dit communement que qui plus hault monte qu'il ne doit, de plus hault tombe qu'il ne vouldroit, et n'est riens plus desplaisant a Dieu qu'orgueil ne plus nuysable a l'omme; mais qui a Deu servyr entend, a la fin son loyer attend (ff. 34-35)' ou lors du châtiment que lui inflige Robert :

et ainsi le fist mourir de male mort, parquoy l'on peut cognoistre que c'est grant folie de desirer chose qui n'apartient a soy d'avoir (fol. 43) ${ }^{7}$.

Le dérimeur n'en appelle pas seulement à la doxa impersonnelle, il invite parfois ses lecteurs à porter eux-mêmes un jugement, en particulier pour souligner le contraste entre le passé du héros (son existence mauvaise et débauchée) et sa pénitence (l'acceptation des pires humiliations):

or pensés quelle vertu de pacience il y avoit en Robert, car celluy qui par avant avoit acoustumé de coucher en lit mol et bien encortiné et en lincieulx [f]ins et delyez en chambre bien parée et tappissée, et avoit acostumé de boyre vins aussy breuvaiges delicieux et manger de toutes bonnes exquises, ainsi comme a son estat apartenoit, il estoit venu a tant qu'il luy failloit boyre et manger, coucher et lever avec les chiens... (fol. 24).

Des commentaires pathétiques visent à entraîner la compassion des lecteurs :

helas, quelle douleur devoit avoir Robert quant il estoit contraint de telles choses souffrir et endurer! (ibid.)

Déjà, dans le récit des males enfances, l'auteur s'apitoyait sur les victimes de Robert, venus se plaindre auprès du duc de Normandie :

et estoit chose piteuse de ouy[r] racompter les grans maulx que Robert fasoit a chescun sans nul espargner (fol. 10).

16 La pénitence du héros donne lieu à des sentences latines (quia ignominia virtutem acuit, fol. 24) ou à des vœux pieux («Dieu y ait part », fol. 22), destinés à suggérer, là encore, l'investissement du narrateur dans l'histoire qu'il raconte.

Motivée par des impératifs pragmatiques, l'écriture en prose n'est pas pour autant dépourvue d'ornements rhétoriques; au contraire, la consommation silencieuse et privée du texte permet au lecteur d'apprécier à sa guise un style recherché, quitte à revenir sur certaines phrases trop longues, voire compliquées.

La Vie du terrible Robert le dyable abonde en clichés stylistiques propres à la fin du Moyen Âge. Le texte-source s'en trouve délayé, et la narration, empreinte de formalisme, accède à un registre élevé qui peut paraître déplacé compte tenu du public visé.

$\mathrm{Au} \mathrm{XV}^{\mathrm{e}}$ siècle, l'amplification est à la mode: les dérimages sont plus étoffés que les récits versifiés dont ils s'inspirent. L'allongement des phrases, les additions, semblent 
traduire un effort pour épuiser la richesse du réel. L'exhaustivité va de pair avec la rigueur historique. Toutefois, l'idéal de brièveté, prôné par Cicéron dans la Rhetorica ad Herennium, reste présent à l'esprit des auteurs, soucieux de préserver la clarté de leur discours. L'incunable de 1496 multiplie les formules d'abrègement; le dérimeur s'excuse ainsi d'écourter le développement d'un sujet qui mériterait d'être traité plus en détails, comme le récit d'une bataille ("Et pour abregier, Robert fit tant que...», fol. 32). Ce souci de condenser l'énoncé n'entre pas en contradiction avec la tendance à la prolixité : la notion de brièveté s'attache à la topique, non aux principes formels. Il paraît importun, voire malséant, de s'attarder sur certains motifs qui, trop familiers, lasseraient le lecteur et entraîneraient d'inutiles digressions ; ainsi s'explique le silence du remanieur lors de l'entrée solennelle de la jeune duchesse de Normandie à Rouen, peu après son mariage :

desquelles choses pour cause de briefveté je m'en tais a present pour plustost condescendre au principal de la matiere (fol. 3).

Parfois, ce refus de tout dire participe d'une stratégie hyperbolique. L'indicible compte au nombre des réflexes stylistiques de l'éloge dithyrambique. Le portrait du duc de Normandie rassemble toutes les vertus courtoises, de sorte que leur énumération devient «quasi impossible» (fol. 2).

21 Le formalisme caractérise lui aussi le style de l'époque. Considérée sous cet aspect, l'écriture en prose calque les maniérismes et le cérémonial qui réglementent la vie aristocratique. En effet, l'emphase, le goût du renforcement superfétatoire, ne se rencontrent pas seulement dans les textes, mais envahissent également les habitudes vestimentaires, comme en témoignent les hennins surélevés, marques de l'élégance féminine, ou les souliers à la poulaine, avec leurs interminables pointes rembourrées ${ }^{8}$. Le gothique se traduit par une certaine recherche de l'extravagance, qui va jusqu'à la provocation. Dans la Vie du terrible Robert le dyable, la forme emphatique donne lieu à des tournures superlatives, visant à susciter l'effroi (le repaire des brigands auxquels s'est associé Robert est «le plus perilleux que l'on ne sçauroit penser ne dyre », fol. 12) ou l'admiration (l'ermite qui dicte au héros sa pénitence est présenté comme «le plus preudomme et le plus saint qui soit au jourd'uy sur terre ", fol. 19). La conversion du mal au bien réunit les deux extrêmes :

Robert, lequel avoyt esté [...] le pyre, le plus cruel, le plus terrible et le plus felon qui oncques fut sur terre, plus orguilleux et fier que ne fut oncques lyon, estoit alors le plus doulx, le plus begnin, le plus humble et le plus debonnayre qu'on eust jamais sur terre veu ne sceu, le plus beau et le plus gracieux en tous ses faitz et ditz et aussi belle contenance que jamais eut prince (fol. 20).

Le préfixe "tres" s'accole à n'importe quel terme ( "tresbien", «tresmauvais", «tresgrant»...), conférant un semblant d'intensité à un référent déjà vidé de toute valeur : l'automatisme du procédé accélère l'usure sémantique.

La synonymie caractérise, elle aussi, la forme diffuse. Une notion, une action, donnent rarement lieu à un mot unique, mais plutôt à des schèmes binaires ("avoir et obtenir pardon ", fol. 20 ; " en dormant et en faisant son repos ", fol. 36) ou ternaires, ordonnés selon un rythme progressif, dans l'enchaînement croissant du nombre de syllabes ("sens, raison et entendement», fol.1). Ces polynômes n'apportent pas de précision supplémentaire, mais contribuent plutôt à une impression de redondance, de "boursouflure "9. S'ils signent l'influence des méthodes scolastiques, par le principe de la division à l'intérieur d'une même catégorie logique, ils témoignent surtout d'une 
propension à l'accumulation énumérative, aux séries tautologiques, d'un amour des mots qui donnera, au XVI ${ }^{e}$ siècle, l'occasion d'un véritable jeu de virtuosité avec, par exemple, les interminables séquences de Rabelais ${ }^{10}$.

Une certaine monotonie se dégage de la répétition d'images stéréotypées, qui reviennent invariablement, quel que soit le contexte. L'auteur de la Vie du terrible Robert le dyable affectionne tout particulièrement les comparaisons animalières; à plusieurs reprises, l'ardeur du héros est assimilée à celle d'un loup,

car il menoit tous ces chiens [sarrasins] devant luy comme ung loup fait ung troppeau de brebis (fol. 31).

Son acharnement à éliminer les païens est décrit de la même manière que la frénésie diabolique de ses débuts, où tous le craignaient «ainsi que les brebis craignent les loupz» (fol.13). La grâce de Dieu a permis, par la pénitence, de métamorphoser le «tigre» en «agneau» (ff.21-22). Cette imagerie, alourdie par un certain maniérisme, traduit la volonté de coller à l'ancienne tradition épique.

Mais le sérieux dont se pare le dérimeur l'amène le plus souvent à imiter les formules du Palais et de la Chancellerie : le style "curial " ${ }^{11}$, protocolaire à l'extrême, donne un aspect méthodique à la narration, non sans artifice. Les expressions itératives «ledict ", «dessusdict », apparaissent parfois dans les contextes les plus incongrus, et ce décalage prête à sourire : « ledit os » " dudit chien » (fol. 23)...Quand cette orientation juridique se justifie, comme c'est le cas lors du conseil tenu par le duc de Normandie, elle enchérit sur la version proposée dans le Dit:

«droit si veult et requiert c'on le face banir » (Dit, v. 291);

«ainsi le trouvons nous aux loys et droitz escriptz et aussi raison le veult et se doit faire » (incunable, fol. 12).

Là encore, l'écriture en prose se définit par l'imitation des registres sérieux, qui tente de faire oublier la teneur fictive et récréative du récit.

Le style du remanieur répond donc à la fois aux besoins de la démonstration et aux goûts de son temps. Les modifications apportées au dispositif narratif reflètent, elles aussi, cette double préoccupation.

La narration subit, par rapport à la version du Dit, une modernisation du lexique et de la topique, un réajustement dans la présentation des personnages, et une explicitation systématique dans l'énoncé des faits.

Le dérimeur a nettoyé le texte des clichés vieillis, dont le sens risquait d'échapper à la compréhension de ses lecteurs. Le vocabulaire a besoin d'être rajeuni. Quelques exemples montrent, dans la réécriture de 1496, un effort de transposition. Lorsque le duc de Normandie fait proclamer à travers tout le duché la condamnation de Robert, il emploie, dans le Dit, une expression ambiguë : «c'on le face banir » (v. 291). Le verbe est employé dans son sens premier («annoncer à cri public») qui, sorti d'usage au $\mathrm{XIV}^{\mathrm{e}}$ siècle, restait encore en vigueur en Normandie. Mais à la fin $\mathrm{du}_{\mathrm{XV}}^{\mathrm{e}}$ siècle, un second sens s'est imposé («chasser, exclure d'un lieu»), comme en témoigne la reprise de l'incunable, qui semble accuser une erreur dans la bouche même du héros : «mon pere m'a fait bannyr de tout son pays » (fol. 16). Simple lapsus, puisque la rubrique du chapitre auquel il fait allusion glose clairement le sens originel du texte-source :

Coment le duc de Normandie fit faire commandement par tout son pays que Robert le dyable son filz et aussy tous les larrons brigans et murtriers de son aliance fussent prins et emprisonnez (fol. 11). 
31 (fol. 12). La réécriture supprime toute ambivalence et facilite ainsi la lecture du public lyonnais. Les régionalismes ne sont pas seuls à poser problème. Le «vieil langage françois ", comme l'a déjà révélé Villon dans sa célèbre ballade, compte au nombre des archaïsmes à la fin du Moyen Âge; aussi faut-il traduire «bacheler» (Dit, v. 596) par "escuyer» (fol. 22) et remplacer le verbe «sachier» (Dit, v. 657) par un terme plus usuel, «tyrer» (fol. 25). De nouveaux vocables apparaissent, comme celui de "gallans", dont use Robert avec ironie pour qualifier les messagers de son père (fol.11) puis les sept ermites tombés sous le coup de sa fureur (fol. 14). folie disparaissent dans l'incunable, comme les vêtements déchirés afin de «miex resambler fol» (Dit, v. 578) ou l'attirance pour les fromages (ibid., vv. 580-583) ${ }^{12}$. Ces détails semblent devenus obscurs, ou véhiculent peut-être des connotations trop fantaisistes, des stéréotypes qui parlent encore à l'imaginaire mais qu'un "traicté» digne de ce nom ne saurait accueillir. Le dérimeur prend soin d'effacer toute trace d'intertextualité avec la littérature de fiction. En supprimant la référence à Trubert (Dit, v. 867), auquel les Romains comparaient le héros, en référence au personnage inventé par Douin de Lavesnes, il nettoie la folie du pénitent de toute intention déceptive (le personnage du fabliau incarnant le trompeur trompé) et, en même temps, évite de blesser la susceptibilité d'un public modeste, sinon rural, par une allusion au sauvage, ignorant les manières de la cour ${ }^{13}$.

quelques appels à la connivence du lecteur concernent l'actualité, ou un passé encore récent. Les bandits qui accompagnent Robert dans ses entreprises sanglantes, dépeints en termes vagues dans le Dit (v.303-306), évoquent dans l'incunable les «escorcheurs » de la Guerre de Cent Ans; le héros apparaît comme le «capitaine » de cette triste "compagnye» (fol. 13). Au cours des excentricités qui caractérisent, à Rome, la folie simulée du pénitent, ce n'est plus une juive que celui-ci pousse dans la boue le jour de ses noces (Dit, vv. 665-668), comme l'exigeait l'antisémitisme d'alors, mais une «royne» (fol. 25) qui pourrait bien rappeler, dans l'esprit des Lyonnais, la figure d'Anne de Bretagne, mariée depuis 1491 à Charles VIII, et qui, pendant l'absence du roi retenu en Italie, fit de fréquents séjours dans leur ville.

La présentation des personnages principaux, dans leur fonction narrative, obéit à une plus grande cohérence. Le dérimeur travaille à affiner leur psychologie, reproduit leurs états d'âme, et leur attribue volontiers des commentaires sur leurs propres actions. Le sentiment de la responsabilité individuelle s'exprime à diverses occasions. Une attention spéciale est accordée à la cellule familiale, qui connaît une importance de plus en plus grande à la fin du Moyen Âge. Le duc de Normandie gagne en épaisseur psychologique; il n'est plus le personnage falot que nous présentait le Dit, réduit au rôle de l'époux impuissant. Désormais, par son tempérament colérique, il partage avec la duchesse l'initiative du pacte diabolique. Dans le Dit, celle-ci, remplie d'amertume et de jalousie, accusait Dieu de favoriser la fertilité des femmes du peuple (vv.65-70) ; l'incunable transfère au duc ce moment de révolte (fol. 4), sans doute pour respecter le portrait de l'épouse idéale, admirable de soumission et de "pacience » (ibid.), ce qui ne l'empêchera pas pour autant de tomber dans le piège de la tentation. La contradiction habite ces personnages tourmentés. Leurs sentiments, loin d'exprimer une taxinomie simpliste et manichéenne, expriment, par leur labilité, la complexité de la nature 
humaine. À la fin du récit, la mère coupable, entendant son fils lui raconter les affres de sa pénitence, pleure de pitié (fol.42), alors que le «diteor» montrait la «dame» impassible (v. 972). De même, l'incunable insiste sur le chagrin de Robert à la mort de son père (fol. 41), ou sur l'amitié de la duchesse pour sa bru (fol. 44). Une certaine sensiblerie commence à poindre.

Le même souci de cohérence amène le remanieur à préciser le rôle du sénéchal félon. Désormais, le personnage intervient au carrefour de deux intrigues, l'une sentimentale (la revendication de la main de la princesse), l'autre guerrière (le siège des Sarrasins devant Rome) : c'est pour se venger du refus que lui oppose l'empereur qu'il coalise les forces étrangères aux portes de la ville (fol. 27, 31). Ce rapport logique ne figurait pas dans le Dit, où l'hostilité du sénéchal entraînait une guerre intestine sans lien avec la menace sarrasine (vv. 686-690). Quant à la version originelle contenue dans le roman, elle expliquait l'arrivée des envahisseurs par l'opportunité qu'offrait, à leurs yeux, la désorganisation interne et l'absentéisme du sénéchal, refusant d'assurer la défense de Rome (vv. 1427-1449) . L'incunable colmate les brèches narratives et rattache le rôle du traitre à une tradition évidente, aisément reconnaissable : le sénéchal déloyal. Par son ambition déçue, le personnage s'apparente au sénéchal de Laudine, dans Yvain; son imposture, qui le pousse à s'attribuer un exploit accompli par le héros, ressemble à celle du sénéchal du roi d'Irlande, lorsqu'il prétend avoir tué le dragon en réalité abattu par Tristan; enfin, il entraîne la mort de son maître et périt à son tour, comme Vertiger dans le roman de $M e r l i{ }^{14}$. Son châtiment offre l'occasion d'exploiter un motif épique : sous le coup meurtrier que lui assène Robert, sa cervelle se répand à terre (fol. 43).

Quant au portrait du héros, il répond à une présentation laïcisée plus nette encore que dans le Dit qui, déjà, amorçait l'évolution par rapport au roman. Sa "sainteté » finale (Dit, v. 1008) s'estompe au profit de sa réhabilitation sociale, au sein de la noblesse :

Robert vesquit longuement avec sa femme et saintement et eut bonne renommée et fut prisé et aymé de grans et de petis car il faisoit de chascun bonne justice et tenoit tout son pays en bonne pays (fol. 40) ${ }^{15}$.

Les crimes dont il occupe les premières années de sa vie l'assimilent moins à l'ennemi de Dieu qu'à l'anti-chevalier. L'épisode de l'adoubement porte la marque de cette sécularisation accrue. Dans le Dit, au moment où son père l'intronise dans le monde des guerriers, Robert affirme sa volonté de persécuter l'Église (vv. 205-207), et la répétera lors du tournoi, dans une déclaration hostile aux moines (vv. 227-228). Le dérimeur supprime ces deux déclarations et les remplace par les protestations qu'adresse au duc le « peuple» victime des manquements de Robert à l'égard code chevaleresque (fol. 10). La responsabilité des nobles dans le maintien de l'ordre importe plus que les pulsions démoniaques qui agitent le possédé. L'auteur de l'incunable prend plaisir à ironiser sur l'errance de l'anti-chevalier, toujours occupé à "sercher quelque mal adventure " (fol. 14). Lorsqu'il prend conscience de sa faute, Robert s'associe aux personnages-types de l'exclusion, «juif» ou «ladre » (fol. 15). Ses déviances l'ont rejeté de la collectivité à laquelle le rattachait sa naissance. Lors de sa réinsertion finale, il cumule les deux qualités inhérentes à l'état de chevalerie: la prouesse et l'amour. Là où le Dit le montrait réticent à accepter la main de la princesse, à qui il préfère d'abord la compagnie de l'ermite (vv. 935-936), l'incunable écarte tout fanatisme mystique au profit d'une discrète idylle amoureuse ; Robert ne saurait épouser à contre cœur la fille de l'empereur, qui s'est éprise de lui : 
et pource qu'elle cogneut Robert estre beau et vaillant chevalier et hardy, et avoyt veue sa meniere d'armer et desarmer, elle le mist tant en son cueur que ce fust merveilles et commença fort a l'aymer (fol. 33).

l'enchaînement et l'explication des faits. Un passage peut subir un transfert destiné à l'intégrer plus rigoureusement dans le processus causal. Ainsi le portrait misérabiliste du pénitent, placé dans le Dit en opposition immédiate avec celui du sénéchal orgueilleux (vv. 817-821), dans une digression préjudiciable à la continuité narrative, n'apparaît dans l'incunable qu'au moment où l'empereur et toute la cour débusquent le héros de sa cachette, dans une scène régie par le principe du regard médiatisé (fol. 36). La prose évite les coupures, la discontinuité, en multipliant les chevilles de transition («apres que», «quant», «lors»...). Cette recherche de la fluidité se lit dans la présentation graphique du texte: l'incunable ne sectionne pas les chapitres en alinéas, mais enchaîne directement les faits entre eux. L'observation rigoureuse de la causalité interne au récit entraîne parfois des répétitions inutiles, comme la longue analepse dans laquelle Robert dresse la liste exhaustive de ses péchés devant l'ermite (fol. 19) : le lecteur, qui connaît déjà les «males chevaleries » du héros, n'a pas besoin de ce rappel, que seule justifie la nécessité intra-diégétique d'informer le confesseur. l'ombre par son prédécesseur. Alors que le Dit rattache, de façon allusive, la stérilité du couple de Normandie à la volonté de Dieu (v. 42) ou à une incompatibilité conjugale (vv. 50-56), l'incunable s'étend sur ces raisons, dont il révèle même le côté avantageux :

car aucunes fois c'est grant proffit a l'homme et a la femme de non avoir enfans et seroit plus proffitable aux peres et meres n'avoir jamais engendré que par faulte d'enseignemens eulx et les enfans estre damnés (fol. 3 ).

41 Plus loin, il explique la passivité des sept ermites, qui se laissent abattre par Robert sans lui opposer de résistance alors qu'ils sont «de bel aage » (Dit, v. 320), «fors et puissans » (fol. 14):

mais ilz ne se volurent pas revenger contre luy et souffrirent pour l'onneur de Dieu tout ce qu'il volut faire (fol. 14).

42 À l'opposé, si les acolytes du héros refusent de suivre leur «capitaine » dans la voie du repentir, c'est parce qu'ils sont trop «acoustumés » au mal, qui leur procure tout leur "plaisir » (fol. 18). Les lynchages et les moqueries dont est victime le pénitent à travers les rues de Rome suscitent un commentaire explicatif, mêlé de désapprobation, de la part de l'auteur:

car c'est la coustume de soy ryre plustoust d'une folye que d'une sagesse : Robert avoit plus de gens autour de luy que s'il eut esté bien saige (fol. 22).

Enfin, l'obstination de l'empereur à refuser la main de sa fille au sénéchal obéit à un raisonnement explicite, puisqu'elle vient de la crainte d'une mésalliance :

il eut fait honte à son lignage (fol. 27) .

Ces situations, ces choix, relevaient de l'arbitraire dans le Dit. 
La mise en prose de Robert le diable coïncide avec l'entrée de la légende dans le monde de l'imprimerie. La réécriture a donc partie liée avec l'ambition d'une diffusion élargie. En soulignant la valeur exemplaire du roman par des réflexions morales ou des citations proverbiales, le dérimeur donne à sa matière une portée générale qui l'insère dans le vaste ensemble de la littérature didactique, au détriment de son aspect récréatif, voire de son originalité. Sa lecture scrupuleuse du texte-source en respecte tous les aspects : rares sont les omissions, et l'on observe plutôt un effet de surenchère au niveau stylistique et au niveau dialectique. Le découpage en chapitres, le refus du mystère, la primauté accordée au narratif, aux faits présentés dans leur succession, participent d'un effort de rationalisation qui vise à authentifier la légende, à lui donner les couleurs du réel, ou du vraisemblable.

en prose, qui caractérise déjà notre récit dans le résumé qu'en contiennent les Chroniques de Normandie, a contribué à ancrer dans l'histoire un personnage de fiction. La notoriété de celui-ci profite au tourisme actuel, puisque les visiteurs des ruines de Moulineaux, près de Rouen, sont invités à y voir les vestiges du château de Robert le diable, assimilé au père de Guillaume le Conquérant, Robert $\mathrm{I}^{\mathrm{er}}$ dit le Magnifique, sixième duc de Normandie, qui se rendit célèbre par ses brutalités et partit expier ses fautes en Terre Sainte, où il mourut, en 1035 :

Alors qu'il était atteint de la peste, porté en litière par quatre noirs, il rencontra un Normand à qui il déclara: «Lorsque tu rentreras en Normandie, tu diras à mon peuple que tu as vu le duc Robert, porté en paradis par quatre diables $»^{16}$.

C'est là une autre manière d'expliquer le célèbre surnom... et de rapprocher l'histoire de la légende. Une contamination réciproque s'observe entre les deux. Le cas n'est pas unique : la récupération pseudo-historique de Robert le diable en Normandie ressemble à celle de Gui de Warewick en Angleterre.

\section{NOTES}

1.Robert le diable, éd. E. Löseth, Paris, 1903 (Société des Anciens Textes Français). Exemplum publié dans les Anecdotes historiques, légendes et apologues tirés du recueil d'Etienne de Bourbon, éd. A. Lecoy de La Marche, Paris, 1877 (Société de l'Histoire de France), § 168. Chroniques de Normandie, Paris, Bibliothèque Nationale de France, ms. fr. 5388, ff. 10-11. Miracles de Nostre Dame par personnages, éd. G. Paris et U. Robert, t. 6, Paris, 1881 (Société des Anciens Textes Français), n 33, pp. 2-77. Dit de Robert le diable, éd. K. Breul, Mélanges A. Tobler, Halle, 1895, pp. 464-509.

2.Lyon, Pierre Mareschal et Bernabé Chaussart : incunable conservé à Paris, Bibliothèque Nationale de France, Rés. Y2-712. Deux folios manquent, au milieu du volume. Georges Doutrepont a démontré le rapport de filiation entre le Dit et la mise en prose de 1496 (Les Mises en prose des épopées et des romans chevaleresques du XIV au XVI ${ }^{e}$ siècle, Bruxelles, 1939 (Slatkine reprints, 1969), p. 310).

3.Paris, Bibliothèque Nationale de France, fr. 25516 (XIII ${ }^{\mathrm{e}}$ s.) et fr. 24405 (fin XIV ${ }^{\mathrm{e}}$-début $\mathrm{XV}^{\mathrm{e}} \mathrm{s}$.). D'après un inventaire de 1405 , la bibliothèque des ducs de Bourgogne possédait 
le premier manuscrit (G. Doutrepont, La Littérature française à la cour des ducs de Bourgogne, Paris, Champion, 1909 (Bibliothèque du XVe siècle, 8), pp. 9-10.

4.Sur cette version, nous renvoyons à notre étude « Pour abregier... Robert le diable, du roman au dit ", PRISMA, XIII-2 (L'amplification - ou son inverse ?), 1997, pp. 169-179.

5.Cf. H.-J. Martin, « Culture écrite et culture orale, culture savante et culture populaire dans la France d'ancien régime ", Journal des Savants, 1975, ne 3-4, pp. 225-282.

6.Cf. Dit, vv. 831-835: « Nonpourquant se dit on souvent meinte feie/Que cil qui le bien gaigne a la foiz ne l'a mie./Le seneschal cuida par son grant vantement/Enginier la pucelle, mais on dit bien souvent/Que de ce que fol pense demeure largement. » 7.Cf. Dit, vv. 1004-1005 : « Mais le sage tesmoingne et on le dit pieça,/C'on convoite tel chose dont il sourt grant hontage. »

8.J. Rasmussen, La prose narrative française au XVe siècle. Etude esthétique et stylistique, Munksgaard, Copenhague, 1958, p. 52.

9.A. Lorian, «Pléonasme et périssologie : le récit redondant au $\mathrm{XV}^{\mathrm{e}}$ siècle », Rhétorique et mise en prose au $X V^{e}$ siècle, actes du $6^{\mathrm{e}}$ colloque international sur le Moyen Français (Milan, 4-6 mai 1988) publiés par S. Cigada et A. Slerca, vol. 2, Milan, Vita e Pensiero, 1991, p. 12.

10.J. Rasmussen, op. cit., p. 51. Cf. A. Lorian, Tendances stylistiques dans la prose narrative française du XVIe siècle, Paris, Klincksieck, 1973 (Bibliothèque française et romane, A-26).

11.J. Rasmussen, op. cit., p. 33 sq.

12.Sur ce motif, voir J. Berlioz, « Métaphore, lieux communs et récit exemplaire : les images de la folie simulée dans la Vie du terrible Robert le dyable (1496) ", Symboles de la Renaissance. Arts et langage, vol. 2, Paris, 1982, pp. 89-108 et pp. 231-236.

13.Sur les significations de Trubert, voir P. -Y. Badel, Le sauvage et le sot, Paris, Champion, 1979 (essais).

14.Sur ces exemples, voir Br. Woledge, «Bons vavasseurs et mauvais sénéchaux », Mélanges Rita Lejeune, vol. 2, Gembloux, Duculot, 1969, pp. 1274-1275.

15.Cf. Dit, vv. 1006-1008 : « Robert, qui en s'anfance ot esté moult sauvage,/Vers Dieu et vers son prime fu de si bon corage/Que je croi qu'il conquist des sainz ciex l'eritage. » 16.Paris-Normandie, 17 août 1983.

\section{AUTEUR}

\section{ÉLISABETH GAUCHER}

Université Lille-III Charles-de-Gaulle 